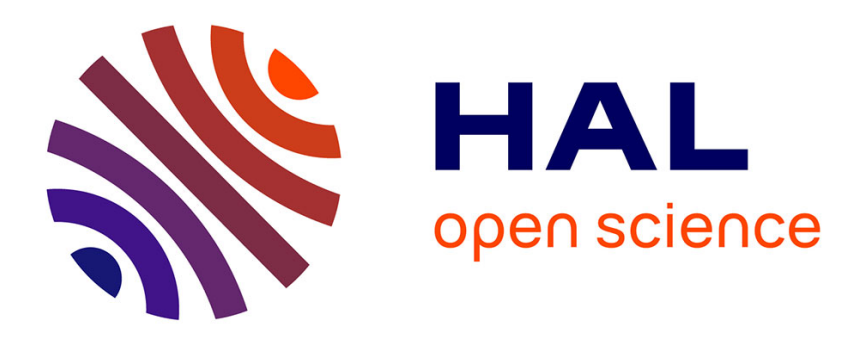

\title{
Effects of collisions on energetic particle-driven chirping bursts
}

Maxime Lesur

\section{To cite this version:}

Maxime Lesur. Effects of collisions on energetic particle-driven chirping bursts. Physics of Plasmas, 2013, 20, pp.055905. 10.1063/1.4804644 . hal-01969744

HAL Id: hal-01969744

https://hal.science/hal-01969744

Submitted on 14 Jan 2019

HAL is a multi-disciplinary open access archive for the deposit and dissemination of scientific research documents, whether they are published or not. The documents may come from teaching and research institutions in France or abroad, or from public or private research centers.
L'archive ouverte pluridisciplinaire HAL, est destinée au dépôt et à la diffusion de documents scientifiques de niveau recherche, publiés ou non, émanant des établissements d'enseignement et de recherche français ou étrangers, des laboratoires publics ou privés. 


\section{AIP Plasmas}

\section{Effects of collisions on energetic particle-driven chirping bursts}

M. Lesur

Citation: Phys. Plasmas 20, 055905 (2013); doi: 10.1063/1.4804644

View online: http://dx.doi.org/10.1063/1.4804644

View Table of Contents: http://pop.aip.org/resource/1/PHPAEN/v20/i5

Published by the American Institute of Physics.

\section{Additional information on Phys. Plasmas}

Journal Homepage: http://pop.aip.org/

Journal Information: http://pop.aip.org/about/about_the_journal

Top downloads: http://pop.aip.org/features/most_downloaded

Information for Authors: http://pop.aip.org/authors

\section{ADVERTISEMENT}

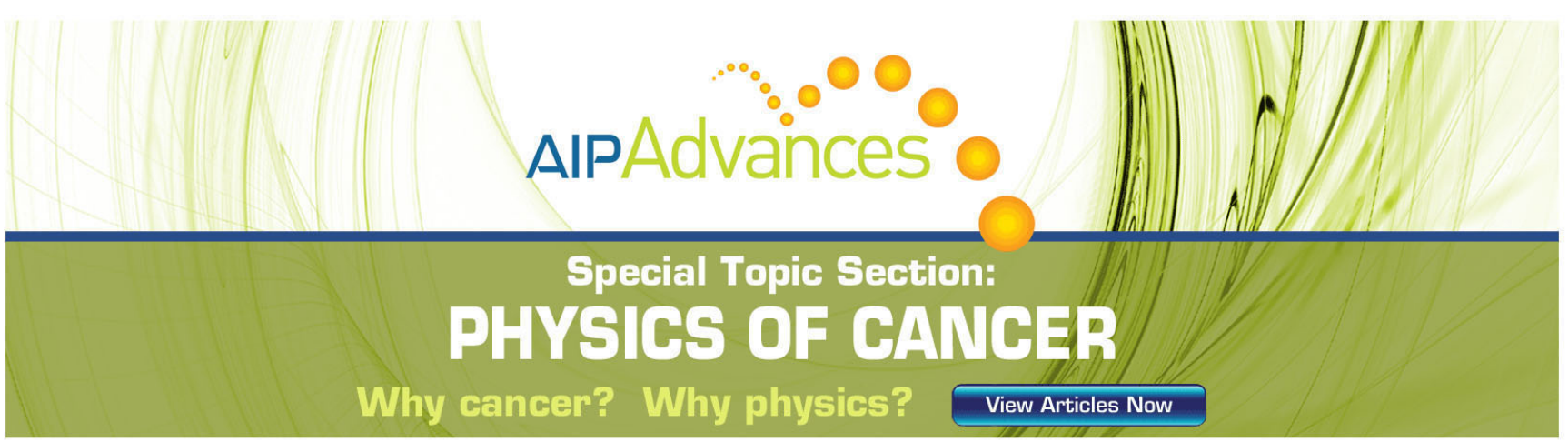




\title{
Effects of collisions on energetic particle-driven chirping bursts ${ }^{a)}$
}

\author{
M. Lesurb) \\ Itoh Research Center for Plasma Turbulence, Kyushu University, Kasuga, Kasuga Koen 6-1, \\ Fukuoka 816-8580, Japan
}

(Received 16 November 2012; accepted 21 March 2013; published online 10 May 2013)

\begin{abstract}
In the presence of an energetic particle population in a dissipative plasma, self-trapped structures in phase-space (holes and clumps) emerge from nonlinear wave-particle interactions. Their dynamics can lead to a nonlinear continuous shifting of the wave frequency (chirping). The effects of collisions on chirping characteristics are investigated, with a one-dimensional kinetic model. Existing analytic theory is extended to account for Krook-like collisions, which quantitatively explains a significant departure from widely accepted square-root time dependency. Relaxation oscillations, associated with chirping bursts, are investigated in the presence of dynamical friction and velocity-diffusion. The period increases with decreasing drag and weakly increases with decreasing diffusion. The mechanism is clarified with a simple semi-analytic model of hole/clump pair, which satisfies a Fokker-Planck equation. The model shows that the linear growth rate cannot be obtained simply by fitting an exponential to the amplitude time-series. (C) 2013 AIP Publishing LLC. [http://dx.doi.org/10.1063/1.4804644]
\end{abstract}

\section{INTRODUCTION}

In an ignited tokamak, the confinement of $\alpha$-particles is critical to prevent damages on the first-wall and to achieve break-even. A major concern is that high energy ions can excite plasma instabilities in the frequency range of Alfvén Eigenmodes (AEs), which significantly enhance their transport. In general, these instabilities are described in a threedimensional (3D) configuration space. However, near the resonant surface, it is possible to obtain a new set of variables in which the plasma is described by a one-dimensional (1D) Hamiltonian in 2 conjugated variables, ${ }^{1-4}$ if we assume an isolated single resonance. In this sense, the problem of AEs is homothetic to a simple 1D single mode bump-on-tail instability. The Berk-Breizman (BB) problem ${ }^{1,2,5}$ is a generalization of the bump-on-tail problem, where a damping term is added in the wave equation with a rate $\gamma_{d}$, to account for background dissipative mechanisms. Observed quantitative similarities between BB nonlinear theory and both global TAE simulations ${ }^{3,6}$ and experiments ${ }^{7-9}$ are an indication of the validity of this reduction of dimensionality.

A feature of the nonlinear evolution of AEs, the frequency sweeping (chirping) of the resonant frequency by $10 \%-30 \%$ on a timescale much faster than the equilibrium evolution, has been observed in the plasma core region of tokamaks JT-60U, ${ }^{10}$ DIII-D, ${ }^{11}$ the Small Tight Aspect Ratio Tokamak (START), ${ }^{12}$ the mega amp spherical tokamak (MAST) ${ }^{6}$ the National Spherical Torus Experiment (NSTX), ${ }^{13}$ and in stellerators such as the Compact Helical Stellerator (CHS). ${ }^{14}$ In general, two branches coexist, with their frequency sweeping downwardly (down-chirping) for one, upwardly (up-chirping) for the other. In most of the

\footnotetext{
a) Paper TI2 4, Bull. Am. Phys. Soc. 57, 292 (2012).

${ }^{\mathrm{b}}$ Invited speaker. Electronic mail: maxime.lesur@polytechnique.org
}

experiments, relaxation oscillations are observed, with quasiperiodical chirping bursts. The period is in the order of the millisecond. Chirping bursts are associated with significant transport of energetic particles, in particular when they trigger avalanches. ${ }^{15}$ This motivates our investigation of the period of chirping bursts.

Qualitatively similar chirping modes are spontaneously generated in the $\mathrm{BB}$ model. Existing theory predicts the time evolution of the frequency shift as $\delta \omega \sim \sqrt{t}{ }^{16}$ In this work, we extend the latter theory by accounting for Krook-like collisions with frequency $\nu_{a}$, which yields $\delta \omega \sim \sqrt{t}\left(1-\nu_{a} t / 3\right)$.

Relaxation oscillations were predicted when collision frequency is small compared to damping rate. ${ }^{17}$ When collisional velocity-space diffusion is small but finite, they were observed in simulations, associated with chirping bursts. ${ }^{18}$ Here, we consider the experimentally relevant regime where these pulsations are quasi-periodic. We clarify the mechanism of relaxation oscillation. We show that the period is mainly dictated by dynamical friction and velocity-space diffusion. Between two bursts, the wave amplitude is low, and collisions dominate over the nonlinear term in the kinetic equation. By modeling a hole and a clump in the velocity distribution by two Gaussians, their dynamics is obtained as the analytic solution of a Fokker-Planck equation, given an initial fit of the structures just after a burst. We obtain a good agreement between analytic prediction and numerical simulation for the hole/clump width, amplitude, and shift. The instantaneous quasi-linear growth rate is then obtained numerically by solving a linear equation system. This procedure recovers time-evolution of amplitude growth and leads to a better qualitative understanding of the nonlinear evolution of wave amplitude between bursts. In addition, our theory explains why the growth rate of the first burst, which is equal to the linear growth rate, is different from (in our simulation, twice larger than) the growth rate of subsequent bursts. 


\section{MODEL}

\section{A. Beam model}

We assume a single electrostatic wave, with a wave number $k$. The assumption that the electric field is sinusoidal corresponds to the situation of a single resonance, which is selected by the geometry in more complex systems. ${ }^{19,20}$ It is appropriate to normalize time to the plasma frequency $\omega_{p}$, distance to the wave number $k$, density to the total plasma density $n_{0}$, and electric field to $m \omega_{p}^{2} /(e k)$, where $e$ and $m$ are the particle charge and mass and $\omega_{p}^{2} \equiv n_{0} e^{2} /\left(\epsilon_{0} m\right)$.

We adopt a perturbative approach and cast the BB model in a reduced form, which describes the time evolution of the beam particles only. ${ }^{21}$ The main hypothesis in this approach is that the bulk particles interact adiabatically with the wave, so that their contribution to the Lagrangian can be expressed as a part of the electric field. In this model, the linear frequency of the wave is imposed as $\omega_{0}=\omega_{p}$. This implies that in practice, time is normalized to the linear frequency of the wave (without nonlinear modification). Even when chirping occurs, $\omega_{0}$ does not change. Chirping (existence of a significant spectral component at finite $\delta \omega$ $\left.\equiv \omega-\omega_{0}\right)$ is due to the nonlinear evolution of the amplitude and phase of the wave. The evolution of the beam distribution, $f(x, v, t)$, is given by a kinetic equation,

$$
\frac{\partial f}{\partial t}+v \frac{\partial f}{\partial x}+\tilde{E} \frac{\partial f}{\partial v}=\mathcal{C}(\delta f)
$$

where $\mathcal{C}(\delta f)$ is a collision operator described below, $f_{0}(v)$ is the initial velocity distribution, $\delta f \equiv f-f_{0}$, and the pseudoelectric field $\tilde{E}$ is defined as

$$
\tilde{E}(x, t) \equiv Q(t) \cos (x-t)-P(t) \sin (x-t) .
$$

The evolution of the pseudo-electric field is given by

$$
\begin{aligned}
\frac{\mathrm{d} Q}{\mathrm{~d} t} & =-\frac{1}{2 \pi} \int f(x, v, t) \cos (x-t) \mathrm{d} x \mathrm{~d} v-\gamma_{d} Q, \\
\frac{\mathrm{d} P}{\mathrm{~d} t} & =+\frac{1}{2 \pi} \int f(x, v, t) \sin (x-t) \mathrm{d} x \mathrm{~d} v-\gamma_{d} P .
\end{aligned}
$$

The term proportional to $\gamma_{d}$ is an external wave damping, which is a model for all linear dissipative mechanisms of the wave energy to the background plasma. ${ }^{21}$ Eqs. (3) and (4) are designed so that $d W / d t=P_{h}-2 \gamma_{d} W$, where $W=\left(Q^{2}+P^{2}\right) / 2$ is the total wave energy, including sloshing energy, and $P_{h}$ is the power the energetic particles transfer to the wave. The bounce frequency of particles deeply trapped into the potential well, $\omega_{b}=\left(Q^{2}+P^{2}\right)^{1 / 4}$, can be used as a measure of the field amplitude.

We define $\gamma_{L 0}$ as a measure of the slope of $f_{0}$ at the resonant velocity,

$$
\left.\gamma_{L 0} \equiv \frac{\pi}{2} \frac{\partial f_{0}}{\partial v}\right|_{v=1}
$$

In the collisionless limit, when $\gamma \ll \omega_{0}$, the linear growth rate reduces to $\gamma=\gamma_{L 0}-\gamma_{d}$. In the initial condition we apply a small perturbation, $f(x, v, t=0)=f_{0}(v)(1+\epsilon \cos k x)$, and the initial values of $Q$ and $P$ are given by solving Poisson's equation.

We consider two collision models. On the one hand, a large part of existing theory takes into account collisions in the form of a Krook operator, ${ }^{22}$

$$
\mathcal{C}_{\mathcal{K}}(\delta f)=-\nu_{a}(\delta f)
$$

which is a simple model for collisional processes that tend to recover the initial distribution at a rate $\nu_{a}$, including both source and sink of energetic particles. On the other hand, a more realistic collision operator, the one-dimensional projection of a Fokker-Plank operator, ${ }^{9,23}$ includes a dynamical friction (drag) term and a velocity-space diffusion term,

$$
\mathcal{C}_{\mathcal{F} \mathcal{P}}(\delta f)=\nu_{f}^{2} \frac{\partial(\delta f)}{\partial v}+\nu_{d}^{3} \frac{\partial^{2}(\delta f)}{\partial v^{2}} .
$$

We refer to the above reduced model as the $\delta f \mathrm{BB}$ model. Compared to the full- $f$ model studied in Ref. 24 , the $\delta f$ model does not take into account effects of the timeevolution of the bulk particle velocity distribution, which are irrelevant to the study of AEs. This assumption is justified by the orders-of-magnitude difference in time-scale between the energetic particle evolution and the evolution of bulk plasma parameters and profiles. In addition, the $\delta f$ model assumes a constant total number of energetic particles and a sine wave structure.

\section{B. Numerical simulation}

In Ref. 24, we described the kinetic code COBBLES (COnservative Berk-Breizman semi-Lagrangian Extended Solver), capable of long-time simulations of the $\delta f \mathrm{BB}$ model with a Krook collision operator. In Ref. 18, COBBLES was extended to include drag and diffusion in the collision operator.

Hereafter, the initial velocity distribution $f_{0}$ is designed with a constant slope in the region where phase-space structures evolve (in a neighborhood of the resonant velocity $v=1$ ). We arbitrarily choose

$$
\frac{\pi f_{0}}{2 \gamma_{L 0}}= \begin{cases}-\exp \left[\sigma^{2}-\left(\frac{v}{0.1}+\sigma\right)^{2}\right] & \text { if } v<0 \\ v-1 & \text { if } 0 \leq v \leq 2 \\ +\exp \left[\sigma^{2}-\left(\frac{v-2}{0.1}+\sigma\right)^{2}\right] & \text { if } v>2,\end{cases}
$$

with $\sigma=0.05$.

\section{EFFECT OF KROOK COLLISION ON CHIRPING VELOCITY}

Ref. 16 shows how one can isolate one spectral component and model it by a Bernstein-Green-Kruskal (BGK) wave to obtain the time-evolution of one chirping event. This theory is based on the following assumptions:

- The resonant velocity of a hole/clump evolves slowly enough for trapped particle orbits to keep their coherency, $\dot{\delta \omega} / \omega_{b}^{2}, \ddot{\delta \omega} / \omega_{b}^{3} \ll 1$; 
- The width of a hole/clump evolves slowly enough for trapped particle orbits to keep their coherency, $\dot{\omega}_{b} / \omega_{b}^{2} \ll 1$

- Holes and clumps are narrow enough that they do not overlay each others, $\omega_{b} / \delta \omega \ll 1$.

With the above assumptions, the perturbation of passing particle distribution is negligible, and a bounce-average treatment of trapped particle distribution yields the frequency shift, in the collisionless limit, as

$$
\delta \omega(t)=\alpha \beta \gamma_{L 0} \sqrt{\gamma_{d} t},
$$

with $\alpha \approx 0.44, \beta=1$; and a saturation level as

$$
\omega_{b} \approx 0.54 \gamma_{L 0} .
$$

In Ref. 16, Eq. (9) is derived by changing variables to action-angle of the bounce-motion of particles trapped in a hole or a clump, and by noting that the unperturbed part $f_{0}(\omega)$ of $f$ does not contribute to the resonant energy exchange. Thus, the wave equation involves only the deviation from the unperturbed distribution at the center of the evolving hole/clump, $g \equiv f-f_{0}\left(\omega_{0}+\delta \omega\right)$. Then, $g$ is expanded in powers of $\epsilon$,

$$
g=g_{0}+\epsilon g_{1}+\ldots
$$

where $\epsilon \equiv \max \left(\ddot{\delta \omega} / \omega_{b}^{3}, \dot{\omega}_{b} / \omega_{b}^{2}, \omega_{b} / \delta \omega\right) \ll 1$, and it is shown that to zeroth order in $\epsilon$, the real and imaginary parts of the wave equation are reduced to

$$
\begin{gathered}
\gamma_{d}=-\frac{2 \gamma_{L 0}}{\pi \omega_{b}^{2} \partial_{\omega} f_{0}\left(\omega_{0}\right)} \int_{0}^{J_{\max }} \frac{\dot{\delta \omega}}{\omega_{b}^{2}} g_{0} \mathrm{~d} J, \\
\delta \omega=-\frac{2 \gamma_{L 0}}{\pi \omega_{b}^{2} \partial_{\omega_{0}} f_{0}\left(\omega_{0}\right)} \int_{0}^{J_{\max }}\langle\cos \psi\rangle g_{0} \mathrm{~d} J
\end{gathered}
$$

where angle brackets indicates a bounce-average, $\psi$ is the spatial coordinate in a frame moving with the hole/clump, $J$ is the bounce-motion action, $J_{\max }=8 \omega_{b} / \pi$, and $g_{0}$ is obtained by bounce-averaging the kinetic equation. Then, in the reference, the collisionless limit is consider, in which case $g_{0}$ is simply

$$
g_{0}(t)=f_{0}\left(\omega_{0}\right)-f_{0}\left(\omega_{0}+\delta \omega\right) .
$$

Eq. (9) follows by assuming a constant gradient for $f_{0}$.

The above theory is valid on a timescale smaller than a collision time. Fig. 1 shows a typical time-evolution of electric field amplitude in the chirping regime (for reference). Fig. 2 shows the corresponding spectrogram of the electric field. Note that the vertical axis is the square of $\delta \omega$. It is clear from this figure that the chirping branches do not follow any square-root law. We test above and following theories on the first chirping branch, since after the first chirping burst, the velocity distribution is modified and the assumption of a constant velocity slope is broken.

In Eq. (9), we introduced a correction parameter $\beta$ to account for all effects that are due to the departure from adiabatic conditions. ${ }^{9}$ One of these effects is the collision-

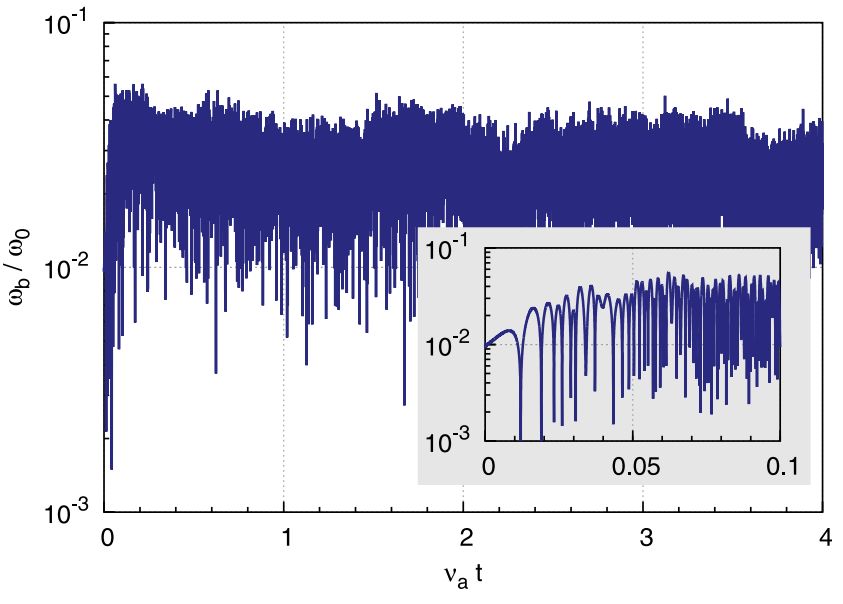

FIG. 1. Time-evolution of the electric field amplitude in $\delta f$-COBBLES simulation with $\gamma_{L 0}=0.05, \gamma_{d}=0.045, \nu_{a}=4 \times 10^{-5}, N_{x} \times N_{v}=128 \times 4096$ grid points, and time-step width $\Delta t=0.05$.

induced trapping of particles near the separatrix,${ }^{26}$ which was observed to account for a $10 \%$ modification $(\beta=1.1)$. The numerical investigation of $\beta$ in Ref. 9 is also limited to a timescale smaller than a collision time. Here the value of $\beta=1.23$ is obtained by linear fit of $\delta \omega^{2}(t)$ for $\delta \omega / \omega_{0}<10 \%$.

In the following, we include the effect of finite collisions, to explain a deviation from square-root law in longer timescales. Lilley described the effect of drag and diffusion in Ref. 27. In this paper, we consider the case where collisions are modeled by a Krook operator. This was first done in Ref. 28 in less details. Then, the bounce-averaged kinetic equation for $g$ at lowest order is

$$
\frac{\partial g_{0}}{\partial t}+\nu_{a} g_{0}=-\frac{\partial f_{0}}{\partial t}
$$

The solution is

$$
\begin{aligned}
g_{0}(t)= & e^{-\nu_{a} t} f_{0}\left(\omega_{0}\right)-f_{0}\left(\omega_{0}+\delta \omega\right) \\
& +\nu_{a} \int_{0}^{t} f_{0}\left[\omega_{0}+\delta \omega\left(t^{\prime}\right)\right] e^{\nu_{a}\left(t^{\prime}-t\right)} \mathrm{d} t^{\prime} .
\end{aligned}
$$

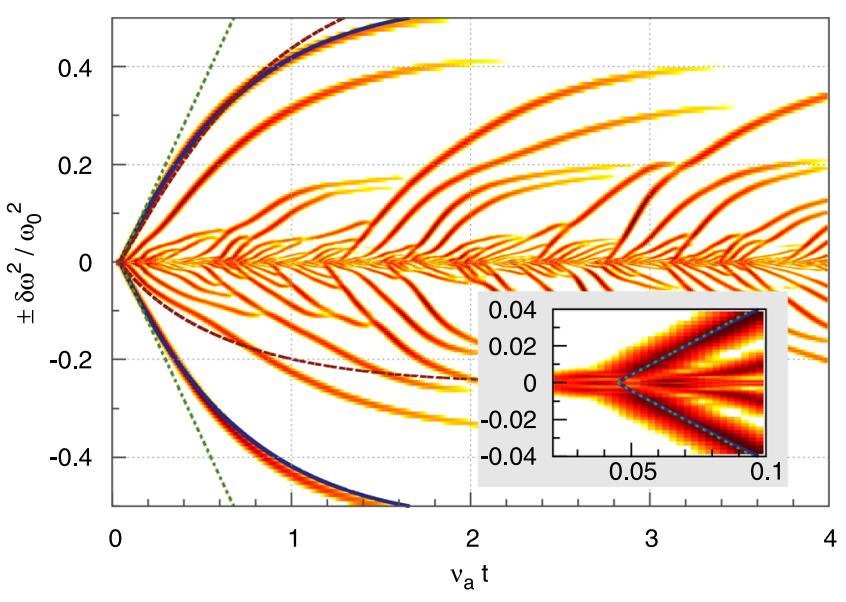

FIG. 2. Effect of finite Krook collisions on chirping velocity. Spectrogram of the electric field, for the simulation shown in Fig. 1. Logarithmic color code ranging from 1 (black) to $10^{-3}$ (white). Two dotted, straight lines correspond to Eq. (9). Two solid curves correspond to Eq. (21). We include a correction coefficient $\beta=1.23$. Two dashed curves correspond to Ref. 25 . Inset: zoom on the beginning of the first chirping event. 
Again, we assume a constant gradient for $f_{0}$,

$$
f_{0}\left[\omega_{0}+\delta \omega(t)\right]=f_{0}\left(\omega_{0}\right)+f_{0}^{\prime}\left(\omega_{0}\right) \delta \omega(t),
$$

which yields

$$
g_{0}(t)=f_{0}^{\prime}\left(\omega_{0}\right)\left[\nu_{a} \int_{0}^{t} e^{\nu_{a}\left(t^{\prime}-t\right)} \delta \omega\left(t^{\prime}\right) \mathrm{d} t^{\prime}-\delta \omega(t)\right] .
$$

We substitute the latter solution into Eqs. (12)-(13) to find, in the limit $\dot{\delta} \omega / \omega_{b}^{2} \ll 1$,

$$
\begin{gathered}
\frac{\gamma_{d}}{\delta \omega}=3 \frac{\dot{\delta \omega}}{\omega_{b}^{2}} \\
\frac{3 \pi^{2} \omega_{b}(t)}{16 \gamma_{L 0}}=1-\frac{\nu_{a}}{\delta \omega(t)} \int_{0}^{t} e^{\nu_{a}\left(t^{\prime}-t\right)} \delta \omega\left(t^{\prime}\right) \mathrm{d} t^{\prime} .
\end{gathered}
$$

We solve the latter equation system by expanding both $\omega_{b}$ and $\delta \omega$ in powers of $\nu_{a} t$. This lengthy but straightforward procedure yields

$$
\begin{aligned}
\delta \omega(t)= & \pm \alpha \beta \gamma_{L 0} \sqrt{\gamma_{d} t}\left[1-\frac{1}{3}\left(\nu_{a} t\right)+\frac{7}{90}\left(\nu_{a} t\right)^{2}\right. \\
& \left.-\frac{19}{1890}\left(\nu_{a} t\right)^{3}+\frac{1507}{1701000}\left(\nu_{a} t\right)^{4}+\ldots\right], \\
\omega_{b}(t)= & \frac{16 \gamma_{L 0}}{3 \pi^{2}}\left[1-\frac{2}{3}\left(\nu_{a} t\right)+\frac{8}{45}\left(\nu_{a} t\right)^{2}\right. \\
& \left.-\frac{8}{315}\left(\nu_{a} t\right)^{3}+\frac{152}{42525}\left(\nu_{a} t\right)^{4}+\ldots\right] .
\end{aligned}
$$

Note that Eqs. (9)-(10) are recovered in the collisionless limit. The effect of finite collision is to reduce the extent of chirping by bending shifting branches. This effect is not negligible since $\delta \omega$ is reduced by $27 \%$ after a collision time, which is of the order of chirping lifetime. Fig. 2 shows a good agreement between Eq. (21) and the observed bended chirping.

In Ref. 25, a top-hat model for holes and clumps was applied to obtain the adiabatic evolution of these idealized structures in the form of two coupled ordinary differential equations for $\delta \omega$ and the structure height $h(t)$ $\equiv \delta f /\left(\omega_{0} \mathrm{~d}_{v} f_{0}\right)$. In the present case of a linear velocity distribution and with our normalization, the system reduces to

$$
\begin{gathered}
\frac{\mathrm{d} h}{\mathrm{~d} t}=-\nu_{a} h-\left(1+\delta \omega / \omega_{0}\right)^{2} h^{2} Q \\
\frac{1}{\omega_{0}} \frac{\mathrm{d} \delta \omega}{\mathrm{d} t}=\left(1+\delta \omega / \omega_{0}\right)^{2} h^{2} Q \\
Q(\delta \omega)=\frac{64}{27 \pi} \frac{M_{1}(\delta \omega)}{M_{2}(\delta \omega)} \gamma_{d} \gamma_{L 0}^{2} \\
M_{1}(\delta \omega)=\frac{1}{2}+\frac{11 \sin 4 \eta}{24 \eta}+8 \cos ^{2} \eta-\left(\frac{3 \sin 2 \eta}{2 \eta}\right)^{2}-\frac{2 \sin 2 \eta}{3 \eta}
\end{gathered}
$$

$$
M_{2}(\delta \omega)=\cos ^{3} \eta(\sin \eta-\eta \cos \eta)
$$

where $\eta=\pi /(2+2 \delta \omega)$. The initial condition (at $t=0)$ is $\delta \omega=\epsilon$ and $h=-\epsilon / \omega_{0}$. Two solutions, which correspond to positive or negative $\epsilon$, with $\epsilon=10^{-3}$, are plotted as dashed curves in Fig. 2 for comparison (without any correction coefficient). The agreement with simulation is not as good as with our theory, especially for the clump. However, our model assumes a sinusoidal mode structure, in contrast with the reference, where the mode structure evolves selfconsistently. To check if the discrepancy is due to this assumption, we run a simulation of the full- $f$ BB model, which is described for example in Ref. 24. In this model, both thermal and beam components of the distribution are evolved kinetically, and nothing is assumed for the mode structure. We design the velocity distribution such that it has a constant slope for $0.5<v<1.95$, with $\gamma_{L 0}=0.05$. The thermal component is a Maxwellian with a thermal velocity 0.1 and density $0.95 n_{0}$. Fig. 3 shows the spectrogram of the electric field. For the down-chirping branch, Nyqvist's theory is, as expected, in better agreement than our sinestructure theory. Nevertheless, there is a significant $50 \%$ discrepancy after one collision time $\left(\nu_{a} t=1\right)$, which remains to be accounted for. For the up-chirping branch, the theory of Ref. 25 is in qualitative disagreement with the model, since the correction from the $\sqrt{t}$ law is in the opposite direction. This disagreement may be due to clump-clump interactions, which are not accounted for in Nyqvist's theory. Indeed down-chirping clumps do not seem to separate well from each others, and their long-range evolution seems to be affected.

\section{EFFECT OF DRAG AND DIFFUSION ON CHIRPING PERIOD}

A regime of quasi-periodic chirping bursts was discovered in Ref. 9 and shown to be relevant to toroidal Alfvén eigenmodes in tokamaks. This regime appears when the

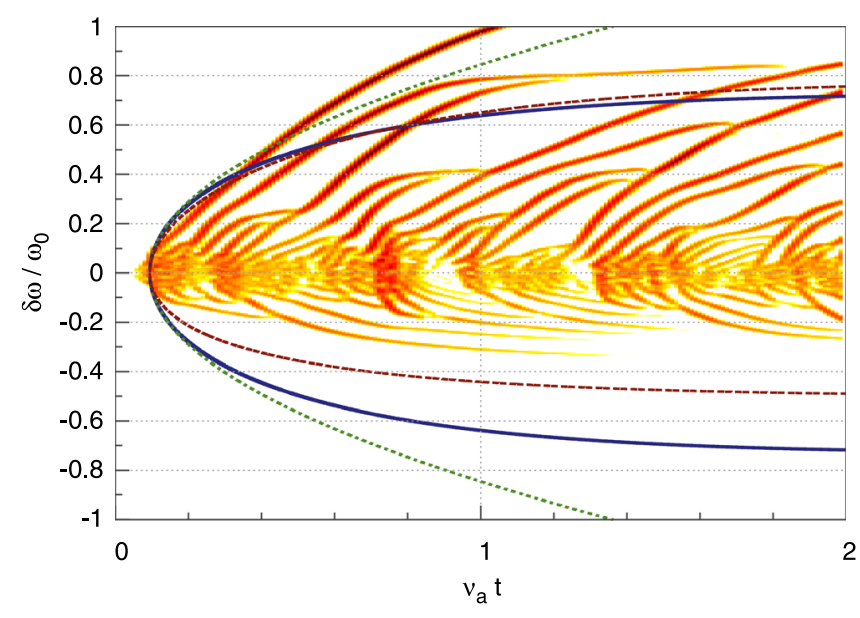

FIG. 3. Effect of finite Krook collisions on chirping velocity, when the mode structure is evolving. Spectrogram of the electric field, for a full- $f$ simulation. Although the model is different, the parameters are the same as for Figs. 1 and 2. Two dotted curves correspond to Eq. (9). Two solid curves correspond to Eq. (21). We include a correction coefficient $\beta=1.23$. Two dashed curves correspond to Ref. 25 . 


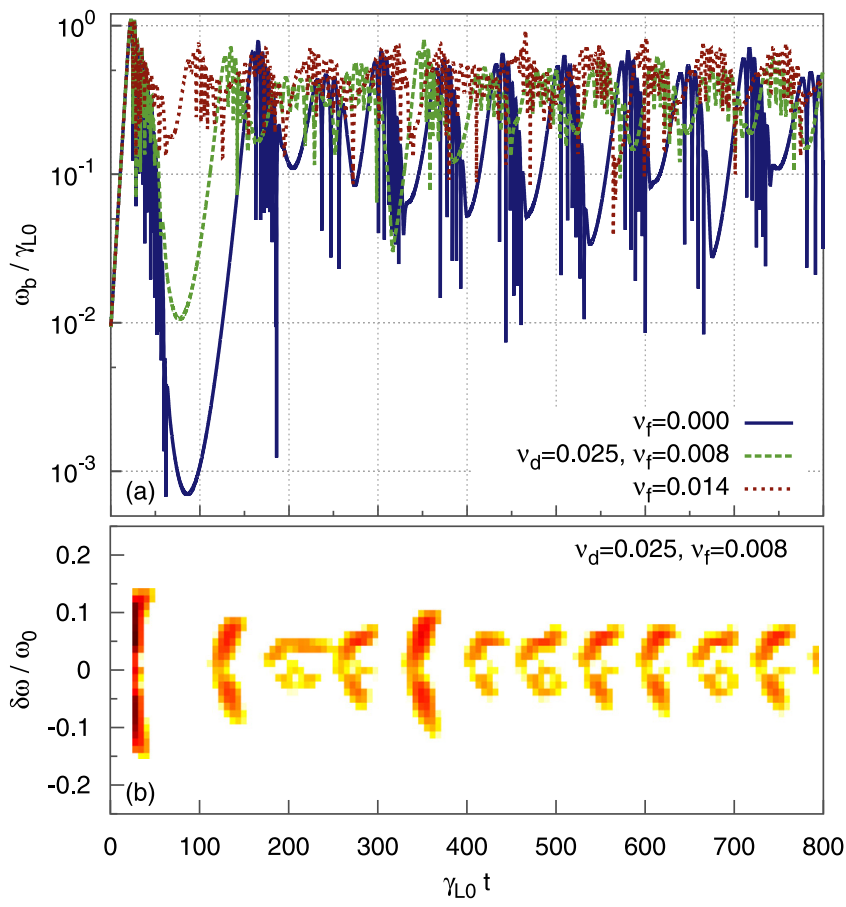

FIG. 4. Effect of drag in the periodic chirping regime. (a) Time-evolution of the electric field amplitude for $\nu_{d}=0.025$ and $\nu_{f}$ shown in the legend. (b) Spectrogram of the electric field, for $\nu_{f}=0.008$. Logarithmic color code ranging from 1 (black) to $e^{-3}$ (white).

drag/diffusion collision operator is applied, for $\nu_{f} \ll \nu_{d}$ $\ll \gamma_{L 0}$ and $\gamma_{d} \approx \gamma_{L 0} / 2$. In this section, we focus on the chirping period $\Delta t_{\text {chirp}}$, which is defined as the average time between two bursts.

Throughout this section, simulation parameters are $\gamma_{L 0}=0.1, \gamma_{d}=0.05, N_{x} \times N_{v}=128 \times 2048$ grid points, time-step width $\Delta t=0.05$. The collision frequencies vary, but we choose a reference case as $\nu_{f}=0.008$ and $\nu_{d}=0.025$. Fig. 4 shows the time-evolution of electric field amplitude for three quasi-periodic chirping with three values of drag, as well as the spectrogram for the reference case, illustrating the quasi-periodic chirping regime. As $\nu_{f}$ increases, $\Delta t_{\text {chirp }}$ decreases. Fig. 5 shows three values of $\nu_{d}$. As $\nu_{d}$ increases, $\Delta t_{\text {chirp }}$ decreases.

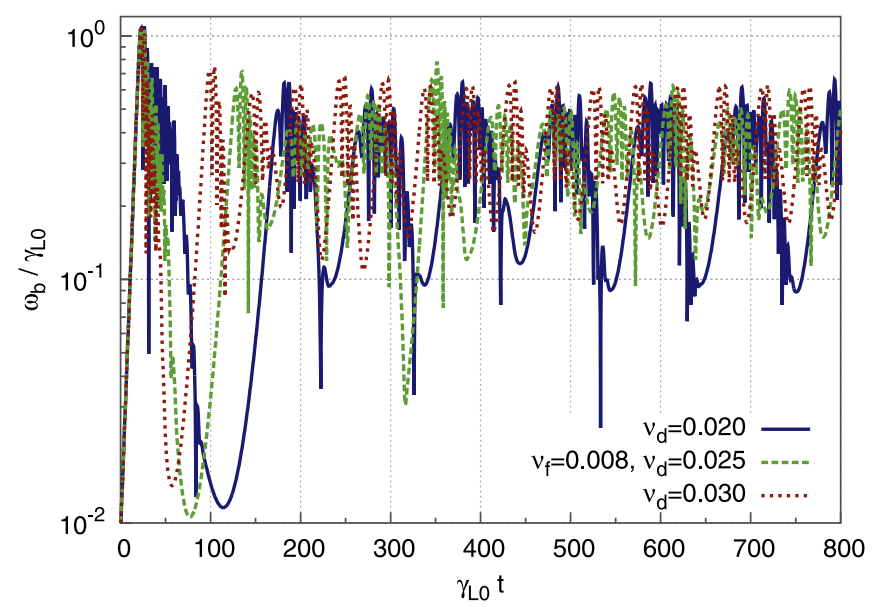

FIG. 5. Effect of diffusion in the periodic chirping regime. Time-evolution of the electric field amplitude for $\nu_{f}=0.008$ and $\nu_{d}$ shown in the legend.
The dependency of chirping period on the model parameters $\left(\gamma_{L 0}, \gamma_{d}, \nu_{f}\right.$, and $\left.\nu_{d}\right)$ is complex and lack theoretical analysis. In the following, we estimate the effects of $\nu_{f}$ and $\nu_{d}$.

\section{A. Gaussian model}

Dupree showed that a phase-space density hole corresponds to a state of maximum entropy, and that this state is a BGK mode, with $\delta f \sim \exp -H / H_{0}$, where $H$ is the energy in the rest-frame of the hole and $H_{0}$ is a constant. ${ }^{29}$ In the periodic chirping regime, the phase-space is dominated by a single hole and a single clump for most of the duration of a chirping burst (except at the very beginning of a burst), as hinted by the observation of a single downwardly shifting branch and a single upwardly shifting branch for each burst in Fig. 4(b). Then the spatial average of the perturbed distribution can be reasonably modeled by two Gaussian distributions,

$$
\begin{aligned}
\langle\delta f\rangle(v, t)= & l_{c} \exp \left[-\left(\frac{v-v_{R}+v_{c}}{\Delta v_{c}}\right)^{2}\right] \\
& -l_{h} \exp \left[-\left(\frac{v-v_{R}-v_{h}}{\Delta v_{h}}\right)^{2}\right],
\end{aligned}
$$

where $l_{c}, l_{h}, v_{c}, v_{h}, \Delta v_{c}$, and $\Delta v_{h}$ are positive functions of time, and the subscripts $c$ and $h$ denote the clump and the hole, respectively. Fig. 6 shows the perturbed velocity distribution for the reference case, at $\gamma_{L 0} t=100$, where $\omega_{b} / \gamma_{L 0} \sim 0.1$. The model is a good fit for the simulation in this particular snapshot. The agreement is typically better for lower amplitudes or just before a burst; worst for higher amplitudes or during and just after a burst.

When the field amplitude is small enough, the collision operator dominates over the nonlinearity $\tilde{E} \partial_{v} f$ in Eq. (1). Then the velocity distribution satisfies a Fokker-Planck equation,

$$
\frac{\partial\langle\delta f\rangle}{\partial t}=\nu_{f}^{2} \frac{\partial\langle\delta f\rangle}{\partial v}+\nu_{d}^{3} \frac{\partial^{2}\langle\delta f\rangle}{\partial v^{2}} .
$$

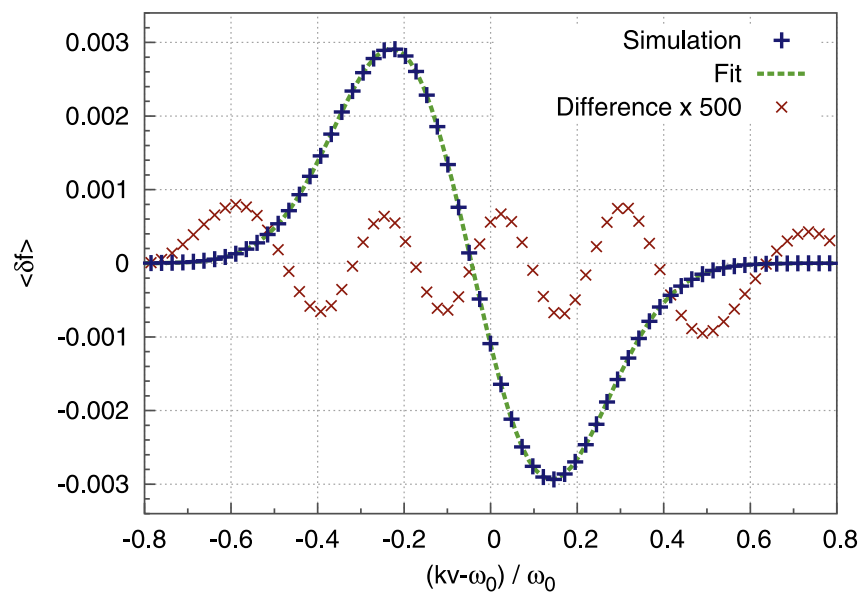

FIG. 6. Perturbed velocity distribution for $\nu_{f}=0.008, \nu_{d}=0.025$, at $\gamma_{L 0} t=100$, and fit, Eq. (28), with $l_{c}=3.20 \times 10^{-3}, l_{h}=3.23 \times 10^{-3}$, $v_{c}=0.203, v_{h}=0.115, \Delta v_{c}=0.215$, and $\Delta v_{h}=0.213$. The difference between the numerical distribution and the fit is amplified 500 times. 
The form of the hole/clump pair is unchanged, and the timeevolution is solved analytically,

$$
\begin{gathered}
l_{c}(t)=\frac{\Delta v_{c}\left(t_{0}\right)}{\Delta v_{c}(t)} l_{c}\left(t_{0}\right), \\
l_{h}(t)=\frac{\Delta v_{h}\left(t_{0}\right)}{\Delta v_{h}(t)} l_{h}\left(t_{0}\right), \\
v_{c}(t)=v_{c}\left(t_{0}\right)+\nu_{f}^{2}\left(t-t_{0}\right), \\
v_{h}(t)=v_{h}\left(t_{0}\right)-\nu_{f}^{2}\left(t-t_{0}\right), \\
\Delta v_{c}(t)^{2}=4 \nu_{d}^{3}\left(t-t_{0}\right)+\Delta v_{c}\left(t_{0}\right)^{2}, \\
\Delta v_{h}(t)^{2}=4 \nu_{d}^{3}\left(t-t_{0}\right)+\Delta v_{h}\left(t_{0}\right)^{2} .
\end{gathered}
$$

Fig. 7 shows the evolution of the hole/clump pair characteristics. We adopt a least-square fit of the hole/clump pair in the simulation at $t=t_{0}$. We arbitrarily choose $\gamma_{L 0} t_{0}=100$ and confirm that the theory is in good agreement with simulation for $40<\gamma_{L 0} t<120$. Similar results are found for various simulation parameters and various choices of $t_{0}$. Observed deviations from a Gaussian distribution do not have qualitative impacts on the evolution of amplitude, shift or width. This justifies that we use the Gaussian model to demonstrate following qualitative statements without lack of generality.

For the sake of explanations, we separate two phases. In the bursting phase, which immediately follows the amplitude saturation, the dynamics is dominated by hole/clump formation. The hole (clump) is rapidly growing and rapidly accelerating (decelerating). Two examples for the reference case
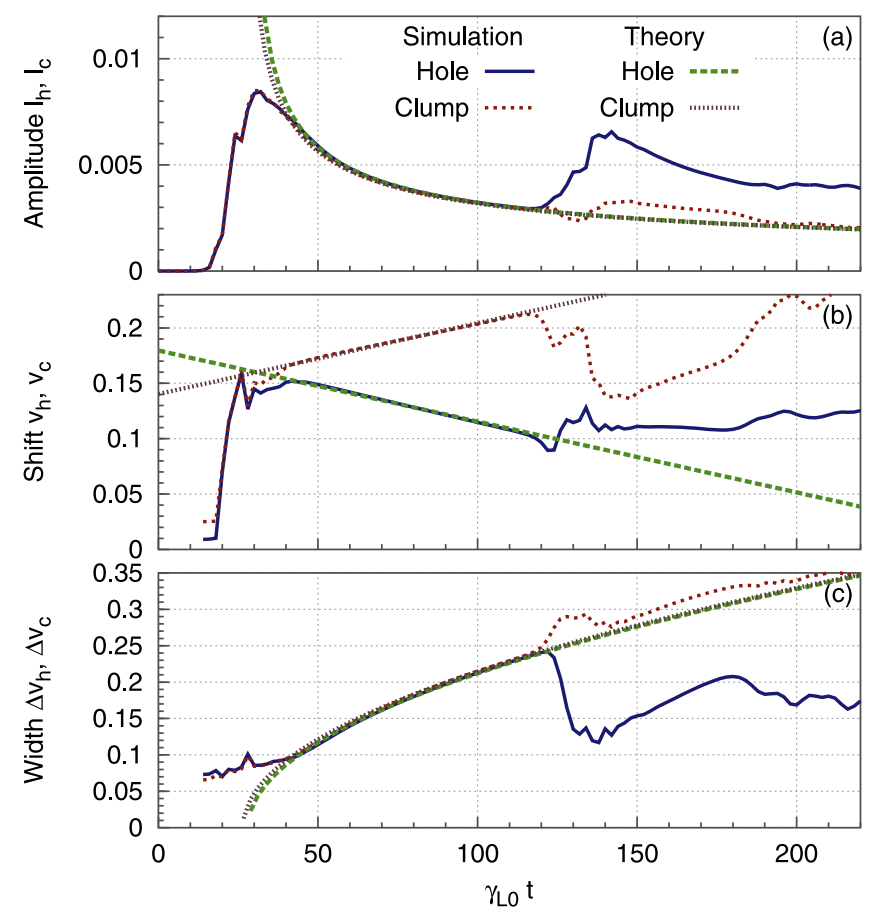

FIG. 7. Time-evolution of hole and clump characteristics, for our reference case $\left(\nu_{f}=0.008, \nu_{d}=0.025\right)$. (a) Amplitude $l_{h}$ and $l_{c}$. (b) Shift $v_{h}$ and $v_{c}$. (c) Width $\Delta v_{h}$ and $\Delta v_{c}$. The dashed curves correspond to Eqs. (30)-(35), with $\gamma_{L 0} t_{0}=100$. are $20<\gamma_{L 0} t<30$ and $120<\gamma_{L 0} t<140$. This phase appears as strong bursts of activity in the spectrogram, Fig. 4(b). In the quiescent phase, the dynamics is dominated by the evolution of one hole and one clump under the effect of dynamical drag and velocity-diffusion. Both hole and clump are slowly decaying and slowly decelerating (for finite $\nu_{f}$ ). Two examples for the reference case are $40<\gamma_{L 0} t<120$ and $140<\gamma_{L 0} t$ $<180$. This phase appears as a quiescent period in the spectrogram. Although we consider two phases for the sake of the explanations below, there is, in fact, no clear separation between these two phases. It should be kept in mind that velocity-diffusion is necessary for the quiescent phase to exist, as was shown in Ref. 9. When collision frequencies are large enough, the initial distribution recovers before the end of the bursting phase, in other word there is no quiescent phase and we leave the periodic chirping regime.

\section{B. Quasi-linear growth rate}

The quasi-linear growth rate is obtained here by using at each instant the Gaussian model of hole/clump pair for the relaxing velocity distribution, and substituting it into the linearized model equations. As a preliminary step to the analysis below, we show that this quasi-linear growth rate reproduces the instantaneous growth rate of wave amplitude measured directly in simulations. To obtain the linear growth rate, we search for solutions of the form $\exp (p t)$, where $p \equiv \gamma-\imath \omega$. Writing $f_{k}(v, t)=f_{p}(v) e^{p t}$ the Fourier components of $\delta f$, and $\exp (-t t)(Q+\imath P)=Z_{p} e^{p t}$, we obtain a linear equation system,

$$
\begin{gathered}
(p+\imath) f_{p}+\frac{Z_{p}}{2} \frac{\partial f_{0}}{\partial v}=\nu_{f}^{2} \frac{\partial f_{p}}{\partial v}+\nu_{d}^{3} \frac{\partial^{2} f_{p}}{\partial v^{2}}, \\
\left(p+\gamma_{d}+\imath\right) Z_{p}=-\int f_{p} \mathrm{~d} v .
\end{gathered}
$$

Discretizing the velocity space as $v_{j}=j \Delta v$ for $j=1 \cdots N_{v}$, the latter system is approximated to first order accuracy in $\Delta v$,

$$
\begin{aligned}
\left(p+\imath v_{j}\right) f_{j}+\frac{Z_{p}}{2} \frac{\partial f_{0}}{\partial v}\left(v_{j}\right)= & \frac{\nu_{f}^{2}}{2 \Delta v}\left(f_{j+1}-f_{j-1}\right) \\
& +\frac{\nu_{d}^{3}}{\Delta v^{2}}\left(f_{j+1}-2 f_{j}+f_{j-1}\right), \\
\left(p+\gamma_{d}+\imath\right) Z_{p} & =-\Delta v \sum_{j=1}^{N_{v}} f_{j},
\end{aligned}
$$

where $f_{j} \equiv f_{p}\left(v_{j}\right)$ and boundary conditions are $f_{0}=f_{N_{v}+1}=0$. This system of $N_{v}+1$ equations is put in the form of an eigenvalue problem and solved using Lapack library. For our reference case in the absence of holes and clumps $\left(l_{c}=l_{h}=0\right)$, we obtain $\omega=1.000$ and $\gamma=0.04476$. In the presence of holes and clumps, we denote the growth rate as $\gamma_{Q L}$, indicating that we use the velocity distribution that results from nonlinear calculations.

From the amplitude time-series of the numerical simulation, we extract the instantaneous growth rate $\gamma_{N L}$, defined as $E\left(t_{2}\right)=E\left(t_{1}\right) \exp \left[\gamma_{N L}\left(t_{2}-t_{1}\right)\right], \quad$ where $\quad \gamma_{L 0}\left(t_{2}-t_{1}\right)=4$. 
Fig. 8 shows that the quasi-linear growth rate obtained from the eigenvalue problem with the Gaussian model for hole and clump is in good agreement with the nonlinear growth rate extracted from the simulation. We could easily go one step further, and use the numerical velocity distribution itself, retrieved from numerical simulations, without modeling it. We would get even better agreement between the quasi-linear and the nonlinear growth rate. However, the goal here is to clarify hole/clump dynamics. To reach this goal, it is necessary to keep models as simple and analytic as reasonably possible.

Here we make an important remark. Fig. 7(a) shows that the second burst occurs before the remnant hole-clump pair from the first burst is completely dissipated. Since the initial distribution function is not recovered, there is a discrepancy between the linear growth rate $\gamma=0.04476$ and the maximum growth reached before the second burst at $\gamma_{L 0} t=122, \gamma_{N L}=0.024$. This discrepancy is marked (A) in Fig. 8. In Alfvén wave experiments in magnetic confinement devices, the amplitude time-series of magnetic perturbation looks as though the linear growth rate $\gamma$ can be extracted by fitting an exponential to the signal. Our analysis shows that this procedure, which is used in data analysis (e.g., Ref. 30), can lead to large error (50\% in our case). In other words the growth is not linear in the case of quasi-periodic chirping bursts. For the same reason, successive chirping rates may not reflect the relaxed distribution $\left(f_{0}(v)\right.$ in the collision operator $\mathcal{C}(f-f 0)$ ), but the instantaneous state of the relaxing distribution $(f(x, v, t))$. In addition, since the discrepancy $(A)$ depends on the details of the velocity distribution, theory predicts the timing of the subsequent burst (e.g., at $\gamma_{L 0} t \approx 125$ ), but only qualitatively.

\section{Effect of drag}

The effect of drag on chirping period is complex and depends on other parameters. Fig. 9 shows the period as a function of drag, for two fixed values of diffusion. The data points are shown only for simulations categorized as periodic

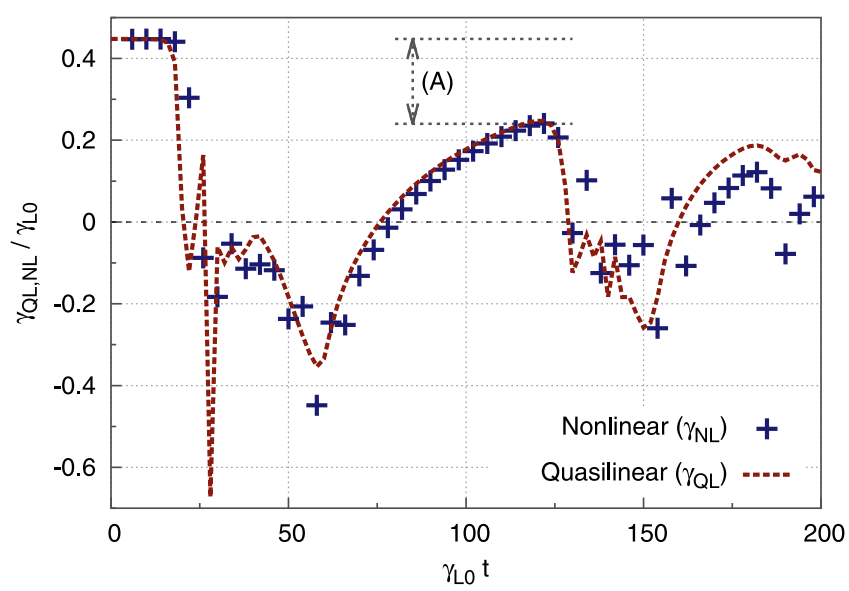

FIG. 8. Time-evolution of the growth rate in our reference case. Points: obtained from the time-series of electric field amplitude (Fig. 5, dashed curve). Dashed curve: obtained from the linearized equations and the Gaussian model for $\partial\langle f\rangle / \partial v$. In the absence of hole and clump, $\gamma / \gamma_{L 0}=0.4476$. (A) shows the discrepancy between the latter value and the maximum growth rate reached before the second burst.



FIG. 9. Effect of drag on chirping period at fixed diffusion, for $\nu_{d}=0.015$ and 0.020 .

chirping, by the categorization algorithm developed in Ref. 18. We observe that, when the period is large, the general trend is a decreasing period as drag increasing. The trend is reversed when the period is small.

To understand this complex behavior, we need to take into account the effect of drag on both bursting phase and quiescent phase. In the bursting phase, drag lengthens the lifetime of the hole by deepening it. ${ }^{27}$ On the one hand, when the bursting phase is significantly shorter than the whole period, this is not a significant effect, as can be seen in Fig. 4(a). Thus the effect of $\nu_{f}$ on chirping period is explained by the effect on the quiescent period. From Eqs. (30)-(35), it is clear that the only effect of drag during the quiescent period is to shift holes and clumps to smaller velocities. After one chirping burst, both hole and clump are roughly shifted by a same amount, here $v_{c 0}=v_{h 0}=0.16$ at $\gamma_{L 0} t=30$, which is the extent of chirping. Fig. 10 shows the nonlinear growth rate against the shift $v_{c}-v_{c 0}=v_{h 0}-v_{h}$,
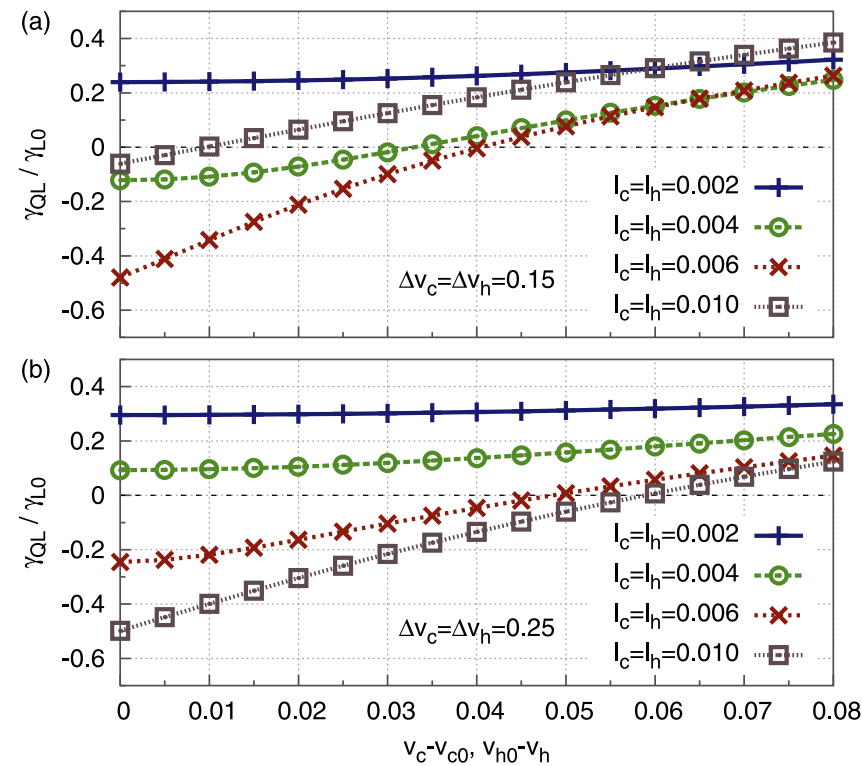

FIG. 10. Effect of hole/clump shift on nonlinear growth rate. (a) Small width $\Delta v_{h}=\Delta v_{c}=0.15$. (b) Large width $\Delta v_{h}=\Delta v_{c}=0.25$. In both cases we choose $v_{c 0}=v_{h 0}=0.16$, where lines cross in Fig. 7(b). 


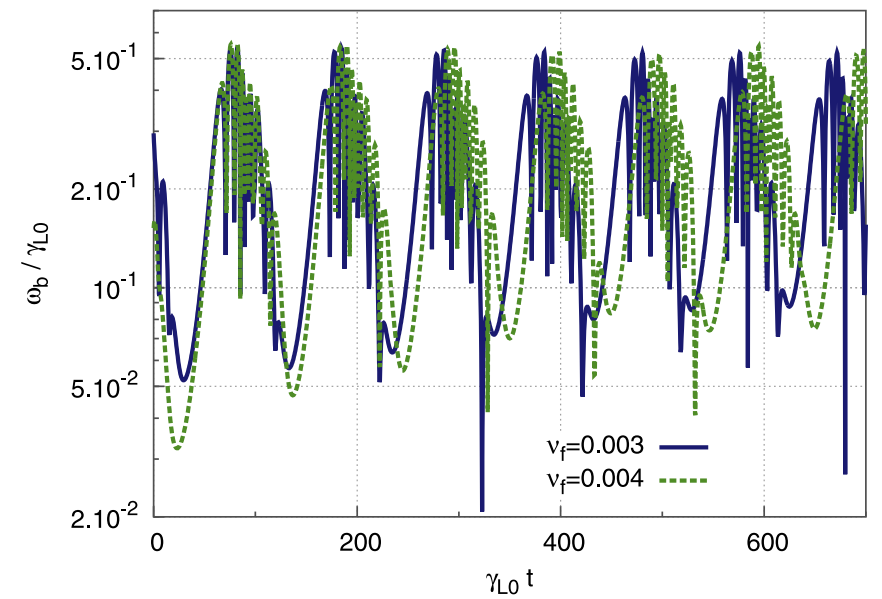

FIG. 11. Special case where chirping period increases with increasing drag. Time-evolution of the electric field amplitude with $\nu_{d}=0.020$ and two values of $\nu_{f}$ shown in the legend. The time is shifted by an arbitrary value, chosen such that the first chirping burst in the plot starts at the same position.

for various hole/clump sizes and widths. We observe that in all these cases, the growth rate increases with shift. This clarifies the effect of drag on period: the larger the drag, the more rapidly the hole/clump pair is decelerated after a burst, the larger the nonlinear growth rate, thus the shorter the quiescent phase and the smaller the chirping period.

On the other hand, when the quiescent phase is much smaller than the whole period, the dominant effect is the lengthening of the bursting phase. In this case, chirping period increases with increasing drag. Fig. 11 shows the timeevolution of the amplitude in such a case.

\section{Effect of diffusion}

The effect of diffusion on chirping period is consistent with simple intuitive arguments. Fig. 12 shows that the period decreases as diffusion increases, for fixed drag. For large diffusion, the effect tends to saturate. Again, the data points are shown only for simulations categorized as periodic chirping.

While the hole/clump amplitude is decreased and the width is increased by diffusion, the product $l_{c, h} \Delta v_{c, h}$ is conserved. Fig. 13 is a scan of the nonlinear growth rate

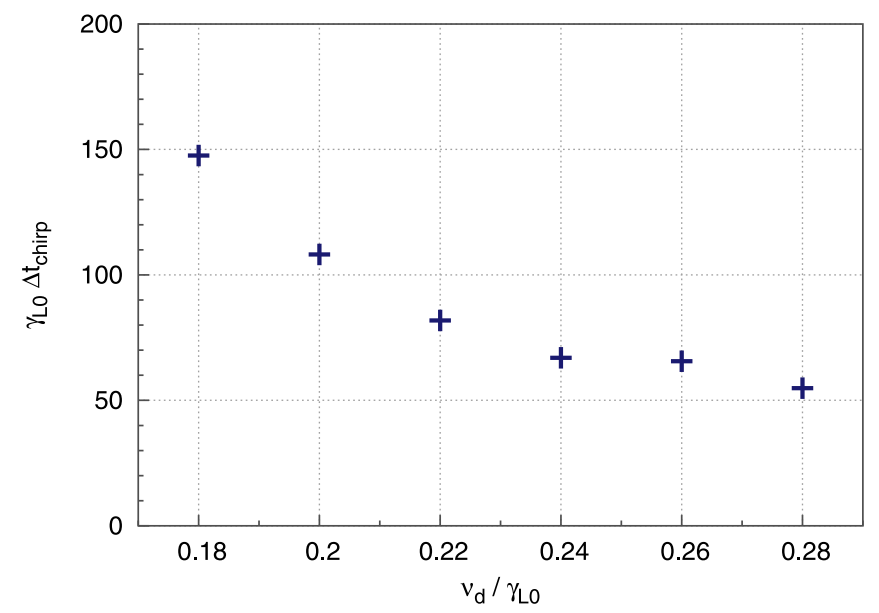

FIG. 12. Effect of diffusion on chirping period at fixed drag, $\nu_{f}=0.005$.

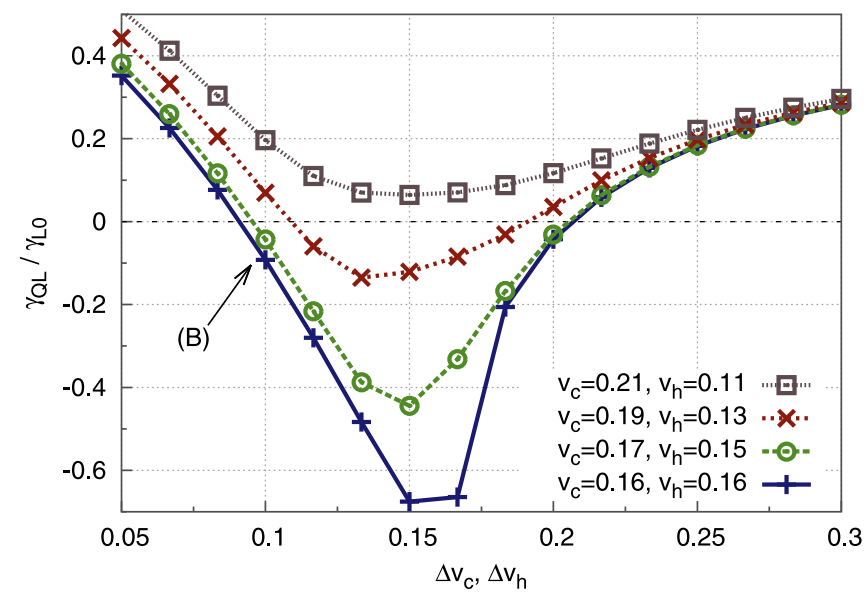

FIG. 13. Effect of diffusion on nonlinear growth rate. The product $l_{c, h} \Delta v_{c, h}=8 \times 10^{-4}$ is kept constant.

for decreasing $l_{c, h}$ and increasing $\Delta v_{c, h}$, while $l_{c, h} \Delta v_{c, h}$ $=8 \times 10^{-4}$ is kept constant, for various shifts of the hole/ clump pair. In all these cases, the rate decreases until $\Delta v_{c, h} \approx 0.15$, then increases. This clarifies the mechanism of relaxation oscillation of the wave amplitude. After a burst, the system is typically at the point marked (B) in Fig. 13 with a negative growth rate. As diffusion acts (as we move to the right in the figure), the growth rate must go through even more negative values before recovering toward the linear growth rate. The larger $\nu_{d}$ is, the quicker this process is, thus the smaller the chirping period.

In addition to the effect of $\nu_{d}$ on the quiescent period, increasing $\nu_{d}$ also decreases the duration of the bursting phase, by damping holes and clumps. This is an additional reason why $\Delta t_{\text {chirp }}$ decreases with increasing $\nu_{d}$.

\section{CONCLUSION}

We have quantified several effects of finite collisions on chirping velocity and chirping period, in the ideal limit of a sine mode structure. Finite Krook collisions bend chirping branches. The discrepancy from square-root time dependency is $27 \%$ after one collision time. In the quiescent phase of the periodic chirping regime, relaxation oscillations are mainly due to diffusion, which brings a hole/clump pair to a shape such that the linearized kinetic equation yields a negative growth rate, before the hole and clump are small enough (in amplitude, large in width) to recover a positive growth rate. The subsequent burst occurs before the velocity distribution completely recovers, leading to instantaneous growth rate about half the initial linear growth rate. Larger values of diffusion shorten the period by combining two effects: (1) structure dissipation during the burst and (2) faster recovery of a positive growth rate. Larger values of drag shorten the period if the quiescent phase is large enough, by deceleration of hole/clump pair, which yields larger nonlinear growth rate. Larger values of drag lengthen the period if the quiescent phase is short, by deepening the hole, which then survives longer.

The accuracy of our simple model of hole/clump pair as two Gaussians is degraded after the first burst, as remnants 
of holes and clumps from former bursts enter the picture. Particle trapping and detrapping are not taken into account in our theory. However, the agreement with simulations, where trapping and detrapping naturally occurs, shows that their effect on hole/clump amplitude, shift, and width is not significant in the quiescent phase. Additional effects, such as holehole or clump-clump interactions, and long-range chirping, ${ }^{31}$ should be taken into account to improve the theory. However, these effects do not impact our qualitative statements when the quiescent phase is larger than the bursting phase, or for the periodic chirping regime with small enough frequency shift. The latter regime is important since it was observed in tokamak experiments. ${ }^{6,10}$ Hole-hole or clumpclump interactions can be neglected because one single hole and one single clump dominate during one chirping burst. In the opposite limit where the duration of the bursting phase is of the same order as the chirping period, a different approach can provide an estimate of the nonlinear growth rate, assuming that the phase-space is dominated by one single structure. $^{32}$

Our results call for promising experiments, where collision frequencies may be used as control parameter of chirping period, to mitigate energetic particle transport in Alfvén wave experiments.

\section{ACKNOWLEDGMENTS}

The author is grateful for stimulating discussions with Y. Idomura, S-I. Itoh, K. Itoh, P. H. Diamond, X. Garbet, Y. Kosuga, and the participants in the 2009 and 2011 Festival de Theorie. This work was supported by a grant-in-aid for scientific research of JSPF, Japan (21224014). Computations were performed on the XT system at RIAM.

${ }^{1}$ H. L. Berk, B. N. Breizman, and M. S. Pekker, Plasma Phys. Rep. 23, 778 (1997).

${ }^{2}$ B. N. Breizman, H. L. Berk, M. S. Pekker, F. Porcelli, G. V. Stupakov, and K. L. Wong, Phys. Plasmas 4, 1559 (1997).

${ }^{3}$ H. V. Wong and H. L. Berk, Phys. Plasmas 5, 2781 (1998).

${ }^{4}$ X. Garbet, G. Dif-Pradalier, C. Nguyen, P. Angelino, Y. Sarazin, V. Grandgirard, P. Ghendrih, and A. Samain, in Proceedings of AIP Conference on Theory of Fusion Plasmas, edited by O. Sauter, X. Garbet, and E. Sindoni (AIP, Melville, NY, ADDRESS, 2008), Vol. 1069, pp. 271-276.
${ }^{5}$ H. L. Berk, B. N. Breizman, and M. Pekker, Phys. Rev. Lett. 76, 1256 (1996).

${ }^{6}$ S. D. Pinches, H. L. Berk, M. P. Gryaznevich, S. E. Sharapov, and the JET-EFDA Contributors, Plasma Phys. Controlled. Fusion 46, S47 (2004).

${ }^{7}$ A. Fasoli, B. N. Breizman, D. Borba, R. F. Heeter, M. S. Pekker, and S. E. Sharapov, Phys. Rev. Lett. 81, 5564 (1998).

${ }^{8}$ R. F. Heeter, A. F. Fasoli, and S. E. Sharapov, Phys. Rev. Lett. 85, 3177 (2000).

${ }^{9}$ M. Lesur, Y. Idomura, K. Shinohara, X. Garbet, and the JT-60 Team, Phys. Plasmas 17, 122311 (2010).

${ }^{10}$ Y. Kusama, G. J. Kramer, H. Kimura, M. Saigusa, T. Ozeki, K. Tobita, T. Oikawa, K. Shinohara, T. Kondoh, M. Moriyama, F. V. Tchernychev, M. Nemoto, A. Morioka, M. Iwase, N. Isei, T. Fujita, S. Takeji, M. Kuriyama, R. Nazikian, G. Y. Fu, K. W. Hill, and C. Z. Cheng, Nucl. Fusion 39, 1837 (1999).

${ }^{11}$ W. W. Heidbrink, Plasma Phys. Controlled. Fusion 37, 937 (1995).

${ }^{12}$ K. G. McClements, M. P. Gryaznevich, S. E. Sharapov, R. J. Akers, L. C. Appel, G. F. Counsell, C. M. Roach, and R. Majeski, Plasma Phys. Controlled. Fusion 41, 661 (1999).

${ }^{13}$ E. D. Fredrickson, N. N. Gorelenkov, and H. L. Berk, Bull. Am. Phys. Soc. 51, 181 (2006).

${ }^{14}$ M. Takechi, K. Toi, S. Takagi, G. Matsunaga, K. Ohkuni, S. Ohdachi, R. Akiyama, D. S. Darrow, A. Fujisawa, M. Gotoh, H. Idei, H. Iguchi, M. Isobe, T. Kondo, M. Kojima, S. Kubo, S. Lee, T. Minami, S. Morita, K. Matsuoka, S. Nishimura, S. Okamura, M. Osakabe, M. Sasao, M. Shimizu, C. Takahashi, K. Tanaka, and Y. Yoshimura, Phys. Rev. Lett. 83, 312 (1999).

${ }^{15}$ S. S. Medley, N. N. Gorelenkov, R. Andre, R. E. Bell, D. S. Darrow, E. D. Fredrickson, S. M. Kaye, B. P. LeBlanc, A. L. Roquemore, and the NSTX Team, Nucl. Fusion 44, 1158 (2004).

${ }^{16}$ H. L. Berk, B. N. Breizman, and N. V. Petviashvili, Phys. Lett. A 234, 213 (1997).

${ }^{17}$ H. L. Berk, B. N. Breizman, and H. Ye, Phys. Rev. Lett. 68, 3563 (1992).

${ }^{18}$ M. Lesur and Y. Idomura, Nucl. Fusion 52, 094004 (2012).

${ }^{19}$ G. Wang, Bull. Am. Phys. Soc. 57, 12 (2012).

${ }^{20}$ H. L. Berk, 24th IAEA Fusion Energy Conference, IAEA/CN-197/TH4-1, San Diego (2012).

${ }^{21}$ H. L. Berk, B. N. Breizman, and M. Pekker, Phys. Plasmas 2, 3007 (1995).

${ }^{22}$ P. Bhatnagar, E. Gross, and M. Krook, Phys. Rev. 94, 511 (1954).

${ }^{23}$ M. K. Lilley, B. N. Breizman, and S. E. Sharapov, Phys. Rev. Lett. 102, 195003 (2009).

${ }^{24}$ M. Lesur, Y. Idomura, and X. Garbet, Phys. Plasmas 16, 092305 (2009).

${ }^{25}$ R. M. Nyqvist, M. K. Lilley, and B. N. Breizman, Nucl. Fusion 52, 094020 (2012).

${ }^{26}$ D. Yu. Eremin and H. L. Berk, Phys. Plasmas 9, 772 (2002).

${ }^{27}$ M. K. Lilley, B. N. Breizman, and S. E. Sharapov, Phys. Plasmas 17, 092305 (2010)

${ }^{28}$ M. Lesur, Ph.D. dissertation, Ecole Polytechnique, France (2011).

${ }^{29}$ T. H. Dupree, Phys. Fluids 25, 277 (1982).

${ }^{30}$ K. Shinohara, M. Takechi, M. Ishikawa, Y. Kusama, A. Morioka, N. Oyama, K. Tobita, T. Ozeki, the JT-60 Team, N. N. Gorelenkov, C. Z. Cheng, G. J. Kramer, and R. Nazikian, Nucl. Fusion 42, 942 (2002).

${ }^{31}$ B. N. Breizman, Nucl. Fusion 50, 084014 (2010).

${ }^{32}$ M. Lesur and P. H. Diamond, Phys. Rev. E 87, 031101 (2013). 